TRANSACTIONS OF THE

AMERICAN MATHEMATICAL SOCIETY

Volume 353, Number 11, Pages 4429-4445

S 0002-9947(01)02823-9

Article electronically published on June 27, 2001

\title{
GAUSS SUMS AND KLOOSTERMAN SUMS OVER RESIDUE RINGS OF ALGEBRAIC INTEGERS
}

\author{
RONALD EVANS
}

Abstract. Let $\mathcal{O}$ denote the ring of integers of an algebraic number field of degree $m$ which is totally and tamely ramified at the prime $p$. Write $\zeta_{q}=$ $\exp (2 \pi i / q)$, where $q=p^{r}$. We evaluate the twisted Kloosterman sum

$$
\sum_{\alpha \in(\mathcal{O} / q \mathcal{O})^{*}} \chi(N(\alpha)) \zeta_{q}^{T(\alpha)+z / N(\alpha)},
$$

where $T$ and $N$ denote trace and norm, and where $\chi$ is a Dirichlet character $(\bmod q)$. This extends results of Salié for $m=1$ and of Yangbo Ye for prime $m$ dividing $p-1$. Our method is based upon our evaluation of the Gauss sum

$$
\sum_{\alpha \in(\mathcal{O} / q \mathcal{O})^{*}} \chi(N(\alpha)) \zeta_{q}^{T(\alpha)}
$$

which extends results of Mauclaire for $m=1$.

\section{InTRODUCTION}

Let $E$ be a field of degree $m$ over $\mathbb{Q}$, and let $\mathcal{O}_{E}$ denote the ring of integers in $E$. Suppose that $p$ is a prime and $\mathfrak{P} \subset \mathcal{O}_{E}$ is a prime ideal such that

$$
p \mathcal{O}_{E}=\mathfrak{P}^{m}, \quad p \nmid m,
$$

that is, $p$ is totally and tamely ramified in $E$. For

$$
q=p^{r}, \quad r \geq 1
$$

consider the finite quotient rings

$$
R_{q}=\mathbb{Z} / q \mathbb{Z}, \quad \mathcal{O}_{q}=\mathcal{O}_{E} / q \mathcal{O}_{E},
$$

which have cardinalities $q$ and $q^{m}$, respectively. For $\alpha \in \mathcal{O}_{E}$ viewed as an element of $\mathcal{O}_{q}$, write $N(\alpha)$ and $T(\alpha)$ to denote the norm and trace of $\alpha$ from $\mathcal{O}_{q}$ to $R_{q}$. For any positive integer $n$, set

$$
\zeta_{n}=\exp (2 \pi i / n) .
$$

For Dirichlet characters $\chi, \eta(\bmod q)$ and $z \in R_{q}^{*}$, define the Gauss sum

$$
G(\chi)=G_{m}(\chi)=\sum_{\alpha \in \mathcal{O}_{q}^{*}} \chi(N(\alpha)) \zeta_{q}^{T(\alpha)}
$$

and the (twisted) Kloosterman sum

$$
K(\eta, z)=K_{m}(\eta, z)=\sum_{\alpha \in \mathcal{O}_{q}^{*}} \eta(N(\alpha)) \zeta_{q}^{T(\alpha)+z / N(\alpha)} .
$$

Received by the editors November 17, 1999 and, in revised form, January 4, 2001.

2000 Mathematics Subject Classification. Primary 11L05, 11T24.

(C)2001 American Mathematical Society 
In the case that $\eta$ is the trivial character, write $\eta=1$ and set

$$
K(z)=K(1, z)
$$

The sums in (1.5) - (1.6) are well-defined, since the summands would be unchanged if a multiple of $q$ were added to $\alpha$.

Mauclaire [9], [10], 2, Theorem 1.6.4, p. 40], Odoni [12], 2] Theorem 1.6.2, p. 33], and Funakura [6], [2, Theorem 1.6.3, p. 37] explicitly evaluated the Gauss sums $G_{1}(\chi)$ for all $r \geq 2$. In $\S 2$ (Theorem 2.2), we extend Mauclaire's results by evaluating the Gauss sums $G_{m}(\chi)$ for all $m$.

Salié [13] evaluated the Kloosterman sums $K_{1}(1, z)$ for all $r \geq 2$. Ye [16] evaluated the Kloosterman sums $K_{m}(1, z)$ in terms of a twisted hyper-Kloosterman sum over $R_{q}^{*}$, in the case that $m$ is prime, $m \mid(p-1)$, and $E / \mathbb{Q}$ is cyclic; see (3.1). In $\S 3$ (Theorem 3.2), we apply Theorem 2.2 to extend Ye's result in the case $r \geq 2$ by evaluating $K_{m}(\eta, z)$ for all $m$ (where $m$ need not be a prime nor a divisor of $p-1$ ). Our evaluations are in terms of twisted hyper-Kloosterman sums over $R_{q}^{*}$ which in turn have been explicitly evaluated in [5]. In Theorem 3.3, we extend Ye's result in the case $r=1$ by evaluating $K_{m}(1, z)$ for all (not necessarily prime) $m$ dividing $p-1$.

In contrast with Ye's determination, we do not require results from local class field theory. Our proof requires only relatively basic results from local and global algebraic number theory.

Ye 18] has pointed out that the results of [16 can be generalized to cyclic extensions $E$ of composite degree $m$ over $\mathbb{Q}$, by applying repeated liftings of prime degree as in Arthur and Clozel [1, Eq. (6.7), p. 60]. For work related to [16] where the prime $p$ is unramified in $E$, see Ye [17]. We note that in both [16] and [17], $E$ is assumed to be cyclic over $\mathbb{Q}$, whereas in this paper, there is no such restriction.

In $\S 4$ (Theorem 4.1), we give a general product formula for the Gauss sums $G_{m}(\chi)$, which reduces in the case $m=r=1$ to the famous Davenport-Hasse product formula [3], 2, Theorem 11.3.5, p. 355] for Gauss sums $(\bmod p)$ given in (3.16).

\section{Evaluation of Gauss sums $G_{m}(\chi)$}

In the case $m=1$, the Gauss sum $G_{m}(\chi)$ over $\mathcal{O}_{q}^{*}$ reduces to the familiar Gauss sum $G_{1}(\chi)$ over $R_{q}^{*}$ defined by

$$
G_{1}(\chi)=\sum_{a \in R_{q}^{*}} \chi(a) \zeta_{q}^{a}
$$

No explicit evaluation of $G_{1}(\chi)$ is known for general $\chi$ in the case $r=1$ (i.e., $q=p$ ), but for $r \geq 2, G_{1}(\chi)$ can be evaluated as follows. We have

$$
G_{1}(\chi)=0 \text { if } \chi \text { is nonprimitive, } r \geq 2
$$


(see [2, Eqs. (1.6.4)-(1.6.5)]). If $r \geq 2$ and $\chi$ is primitive, then

$$
G_{1}(\chi)= \begin{cases}\sqrt{q} \zeta_{q}, & \text { if } r \text { is even, } \\ \sqrt{q} \zeta_{q} \zeta_{8}^{1-p}, & \text { if } p>2 \text { and } r \geq 3 \text { is odd, } \\ \sqrt{q} \zeta_{q} \zeta_{8}, & \text { if } p=2 \text { and } r \geq 5 \text { is odd, } \\ \sqrt{q} \zeta_{q} \zeta_{8}^{-\chi(-1)}, & \text { if } p=2 \text { and } r=3,\end{cases}
$$

provided that $\nu(\chi)=1$, where $\nu=\nu(\chi)$ is defined for $r \geq 2$ by

$$
\begin{gathered}
\chi\left(1+p^{s}\right)=\zeta_{p^{s}}^{-\nu}, \quad \text { for even } r=2 s \geq 2, \\
\left.\chi(5)=(-1)^{\nu}, \quad \text { for } \quad q=8 \quad \text { (i.e., } p=2, r=3\right),
\end{gathered}
$$

and

$$
\chi\left(1+p^{s}+\frac{1}{2} p^{2 s}\right)=\zeta_{p^{s+1}}^{-\nu}, \quad \text { for odd } r=2 s+1 \geq 3, \quad q \neq 8 .
$$

(In $(2.6)$ and in the sequel, $\frac{1}{2}(\bmod p)$ is interpreted as $(p+1) / 2(\bmod p)$ when $p>2$.)

The evaluation of $G_{1}(\chi)$ in $(2.3)$ was proved by Mauclaire [9], [10]. For a shortened proof, see [2, Theorem 1.6.4, p. 40] (where "inner sum on $y$ " should be corrected to read "inner sum on $x$ " in [2, p. 41]).

For $r \geq 2$, the assertion that $\chi$ is primitive is equivalent to the assertion that $p$ does not divide $\nu(\chi)$. When $r \geq 2$ and $\nu(\chi)=1$, the (primitive) character $\chi$ is said to be normalized. When $r \geq 2$ and $\chi$ is primitive but not necessarily normalized, we can evaluate $G_{1}(\chi)$ in terms of a normalized Gauss sum in (2.3), as follows. First write

$$
\chi=\xi^{\nu},
$$

where $\xi$ is a normalized character $(\bmod q)$, and $\nu=\nu(\chi)$ is chosen relatively prime to $q(p-1)$. Then

$$
G_{1}(\chi)=G_{1}\left(\xi^{\nu}\right)=\chi(\nu) \sigma_{\nu}\left(G_{1}(\xi)\right),
$$

where $\sigma_{\nu} \in \operatorname{Gal}\left(\mathbb{Q}\left(\zeta_{q(p-1)}\right) / \mathbb{Q}\right)$ is defined by $\sigma_{\nu}\left(\zeta_{q(p-1)}\right)=\zeta_{q(p-1)}^{\nu}$. Since $G_{1}(\xi)$ is evaluated in (2.3), we see that (2.8) yields an evaluation of $G_{1}(\chi)$ for any primitive character $\chi$, when $r \geq 2$.

In Theorem 2.2 below, we extend the evaluations of $G_{1}(\chi)$ given above by evaluating the Gauss sums $G_{m}(\chi)$ for all $m$. We begin with a lemma which gives a useful representation of the elements of $\mathcal{O}_{q}$. While its proof is $p$-adic, the lemma allows us to prove our main results in the language of global rather than local rings.

Lemma 2.1. There exists $\tau \in \mathcal{O}_{E}$ of degree $m$ over $\mathbb{Q}$ such that

$$
\begin{gathered}
\tau^{m} \equiv p u\left(\bmod q \mathcal{O}_{E}\right) \quad \text { for some integer } u \not \equiv 0(\bmod p), \\
\operatorname{Tr}_{E / \mathbb{Q}}\left(\tau^{i}\right) \equiv 0(\bmod q) \quad(1 \leq i \leq m-1),
\end{gathered}
$$


and

$$
\mathcal{O}_{q}=\left\{\sum_{i=0}^{m-1} \alpha_{i} \tau^{i}: \alpha_{i} \in R_{q}\right\}
$$

Moreover, the $m$ conjugates of $\tau$ over $\mathbb{Q}$ have the form $\tau \zeta_{m}^{i}+q \beta_{i}, 1 \leq i \leq m$, where the $\beta_{i}$ are algebraic integers.

Proof. Choose any $\omega \in \mathcal{O}_{E}$ with $\mathfrak{P} \| \omega$, i.e., $\omega \in \mathfrak{P}-\mathfrak{P}^{2}$. Then the irreducible polynomial of $\omega$ over $\mathbb{Q}$ is $p$-Eisensteinian of degree $m$, and $E=\mathbb{Q}(\omega)$. We also have [11, Theorem 5.5, p. 217] $E_{\mathfrak{P}}=\mathbb{Q}_{p}(\omega)$ and $\left[E_{\mathfrak{P}}: \mathbb{Q}_{p}\right]=m$, where $E_{\mathfrak{P}}$ is the $\mathfrak{P}$-adic completion of $E$, and $\mathbb{Q}_{p}$ denotes the $p$-adic rationals. Let $\mathbb{Z}_{p}$ denote the p-adic integers. By [8, Ex. 13-14, pp. 74, 140] (cf. [15] pp. 324-325]), there exists an element $\pi \in E_{\mathfrak{P}}$ such that

$$
\begin{gathered}
E_{\mathfrak{P}}=\mathbb{Q}_{p}(\pi), \quad \mathcal{O}_{E_{\mathfrak{P}}}=\mathbb{Z}_{p}(\pi), \\
\pi^{m}=p \mu, \quad \text { for some } \mu \in \mathbb{Z}_{p}^{*}, \\
\pi \mathcal{O}_{E_{\mathfrak{P}}}=\mathfrak{P} \mathcal{O}_{E_{\mathfrak{P}}},
\end{gathered}
$$

and

$$
\pi-\omega \in \mathfrak{P}^{2} \mathcal{O}_{E_{\mathfrak{P}}} .
$$

Since $X^{m}-p \mu$ is the irreducible polynomial of $\pi$ over $\mathbb{Q}_{p}$, the $m$ conjugates of $\pi$ over $\mathbb{Q}_{p}$ are $\pi \delta^{j}(0 \leq j \leq m-1)$, where $\delta$ is a primitive $m$-th root of unity in a field extension of $\mathbb{Q}_{p}$. Thus

$$
\operatorname{Tr}_{E_{\mathfrak{P}} / \mathbb{Q}_{p}}\left(\pi^{i}\right)=0, \quad 1 \leq i \leq m-1,
$$

where $\operatorname{Tr}$ denotes the trace. By (2.12)-(2.13), every $\alpha \in \mathcal{O}_{E}$ can be $\pi$-adically represented in the form

$$
\alpha=\sum_{i=0}^{m-1} a_{i} \pi^{i}, \quad a_{i} \in \mathbb{Z}_{p}
$$

We can find $\tau \in \mathcal{O}_{E}$ such that

$$
\tau \equiv \pi\left(\bmod q \mathcal{O}_{E_{\mathfrak{P}}}\right),
$$

by reducing $(\bmod q)$ an appropriate linear combination of $\omega, \omega^{2}, \ldots, \omega^{m-1}$ over $\mathbb{Z}_{p}$. Then $\tau$ has degree $m$ over $\mathbb{Q}$, by the same argument we used to show that $\omega$ has degree $m$ over $\mathbb{Q}$. By (2.13) and (2.18), we see that (2.9) holds for some integer $u$ with $u \equiv \mu\left(\bmod q \mathbb{Z}_{p}\right)$. By $(2.16),(2.18)$ and the fact that

$$
\operatorname{Tr}_{E_{\mathfrak{P}} / \mathbb{Q}_{p}}\left(\tau^{i}\right)=\operatorname{Tr}_{E / \mathbb{Q}}\left(\tau^{i}\right)
$$

11. Corollary, p. 266], we see that (2.10) holds. Equality (2.11) follows easily from (2.17) - (2.18). The last assertion of the lemma results from applying the $m$ different $\mathbb{Q}_{p}$-embeddings of $E_{\mathfrak{P}}$ to both sides of (2.18).

We now evaluate the Gauss sums $G(\chi)=G_{m}(\chi)$ over $\mathcal{O}_{q}^{*}$ in terms of the Gauss sums $G_{1}(\chi)$ over $R_{q}^{*}$ discussed at the beginning of this section. 
Theorem 2.2. If $r=1$, then

$$
G(\chi)=p^{m-1} \bar{\chi}^{m}(m) G_{1}\left(\chi^{m}\right) .
$$

If $r \geq 2$ and $\chi$ is nonprimitive, then $G(\chi)=0$. If $r \geq 2$ and $\chi$ is primitive, then, with $\nu(\chi)$ defined by $(2.4)-(2.6)$,

$$
G(\chi)= \begin{cases}G_{1}(\chi)^{m} p^{(m-1) / 2}\left(\frac{p}{m}\right)^{r}, & \text { if } 2 \nmid m, \\ G_{1}(\chi)^{m} p^{(m-1) / 2} \zeta_{8}^{(1-p)(1-m)}\left(\frac{-D p^{1-m}}{p}\right)^{r+1}\left(\frac{m \nu(\chi)}{p}\right), & \text { if } 2 \mid m,\end{cases}
$$

where $D$ is the discriminant of the number field $E$, and where $G_{1}(\chi)$ is explicitly given by (2.8).

Remark. If $2 \mid m$, then $p>2$ by (1.1). Moreover, $p^{m-1} \| D$ by [11, Theorem 4.8, p. 166]. Hence the Legendre symbols in (2.20) make sense. For a formulation of (2.20) in the case $2 \mid m$ in which $\nu(\chi)$ does not appear, see (2.45).

Proof. For $\alpha \in \mathcal{O}_{q}$, write

$$
\alpha=\sum_{i=0}^{m-1} \alpha_{i} \tau^{i}, \quad \alpha_{i} \in R_{q}
$$

as in (2.11). First suppose that $r=1$, so that $q=p$. Recall the definitions of $T$ and $N$ below (1.3). By Lemma 2.1, $T(\alpha)=m \alpha_{0}$ and $N(\alpha)=\alpha_{0}^{m}$, since $q=p$. Thus

$$
G(\chi)=\sum_{\alpha_{0}, \ldots, \alpha_{m-1} \in R_{p}} \chi\left(\alpha_{0}^{m}\right) \zeta_{p}^{m \alpha_{0}}=p^{m-1} \sum_{a \in R_{p}} \chi^{m}(a) \zeta_{p}^{m a}=p^{m-1} \bar{\chi}^{m}(m) G_{1}\left(\chi^{m}\right),
$$

which proves (2.19).

Suppose now that $r \geq 2$. If $\chi$ is nonprimitive, then $G(\chi)=0$ by an argument analogous to that proving (2.2). Next assume that $\chi$ is primitive. If $m=1$, then (2.20) follows from the definition $(2.1)$ of $G_{1}(\chi)$. Hence assume that $m>1$.

We first prove (2.20) when $\chi$ is normalized. There are three cases.

Case 1: $\nu(\chi)=1, r=2 s, s \geq 1$.

The elements $\alpha \in \mathcal{O}_{q}^{*}$ may be written

$$
\alpha=z+z w p^{s} \quad\left(z \in \mathcal{O}_{p^{s}}^{*}, w \in \mathcal{O}_{p^{s}}\right)
$$

so

$$
G(\chi)=\sum_{z \in \mathcal{O}_{p}^{*}} \chi(N(z)) \zeta_{q}^{T(z)} \sum_{w \in \mathcal{O}_{p^{s}}} \chi\left(N\left(1+w p^{s}\right)\right) \zeta_{p^{s}}^{T(z w)}
$$

Since

$$
N\left(1+w p^{s}\right)=1+T(w) p^{s} \equiv\left(1+p^{s}\right)^{T(w)} \quad(\bmod q),
$$

it follows from the normalization (2.4) that

$$
G(\chi)=\sum_{z} \chi(N(z)) \zeta_{q}^{T(z)} \sum_{w} \zeta_{p^{s}}^{T(w(z-1))}
$$

Using Lemma 2.1, one sees that the inner sum $\sum_{w}$ vanishes unless $z \equiv 1\left(\bmod \tau p^{s-1}\right)$, in which case

$$
\sum_{w}=\operatorname{Card}\left(\mathcal{O}_{p^{s}}\right)=p^{s m}=(\sqrt{q})^{m}
$$


Thus, writing $z=1+x p^{s-1}$ with

$$
x:=\sum_{i=1}^{m-1} x_{i} \tau^{i} \in \mathcal{O}_{p} \quad\left(x_{1}, \ldots, x_{m-1} \in R_{p}\right),
$$

we have

$$
G(\chi)=\left(\sqrt{q} \zeta_{q}\right)^{m} \sum_{x_{1}, \ldots, x_{m-1} \in R_{p}} \chi\left(N\left(1+x p^{s-1}\right)\right) .
$$

Write $N\left(1+x p^{s-1}\right)$ as a product of $m$ conjugates and expand. One sees, using Lemma 2.1, that

$$
N\left(1+x p^{s-1}\right)=1-p^{2 s-1}\left\{\frac{m u}{2} \sum_{i=1}^{m-1} x_{i} x_{m-i}+f\left(x_{1}, \ldots, x_{m-1}\right)\right\},
$$

where $f\left(x_{1}, \ldots, x_{m-1}\right)$ is a $\mathbb{Z}$-linear combination of monomials $x_{i_{1}} \ldots x_{i_{n}}$ with $3 \leq n \leq m, \quad i_{1}+\cdots+i_{n}=m$. If $m=2, f$ is interpreted as 0 . (Note that each coefficient in $f$ is divisible by $p^{s-1}$, so the term $f$ could have been omitted from (2.23) were it not for the pesky case $s=1$.) Since

$$
N\left(1+x p^{s-1}\right)=\left(1+p^{s}\right)^{-p^{s-1}}\left\{\frac{m u}{2} \sum_{i=1}^{m-1} x_{i} x_{m-i}+f\left(x_{1}, \ldots, x_{m-1}\right)\right\},
$$

the normalization (2.4) gives

$$
\chi\left(N\left(1+x p^{s-1}\right)\right)=\zeta_{p}^{\frac{m u}{2}} \sum_{i=1}^{m-1} x_{i} x_{m-i}+f\left(x_{1}, \ldots, x_{m-1}\right) .
$$

Therefore, by (2.22) and (2.3),

$$
G_{1}(\chi)^{-m} G(\chi)=\sum_{x_{1}, \ldots, x_{m-1} \in R_{p}} \zeta_{p}^{\frac{m u}{2}} \sum_{i=1}^{m-1} x_{i} x_{m-i}+f\left(x_{1}, \ldots, x_{m-1}\right) .
$$

Now, $x_{m-1}$ does not actually appear in the polynomial $f\left(x_{1}, \ldots, x_{m-1}\right)$ and so unless $x_{1}=0$, the sum on $x_{m-1}$ in (2.24) vanishes when $m>2$. Therefore we may set $x_{1}=0$ in the summands of $(2.24)$ when $m>2$. Further, $x_{m-2}$ does not appear in the polynomial $f\left(0, x_{2}, \ldots, x_{m-1}\right)$, and so unless $x_{2}=0$, the sum on $x_{m-2}$ vanishes when $m>4$. Continuing in this way, we see that one may set

$$
x_{1}=x_{2}=\cdots=x_{[(m-1) / 2]}=0
$$

in the summands of (2.24). With this substitution, all terms of the polynomial $f$ vanish, and so (2.24) becomes

$$
G_{1}(\chi)^{-m} G(\chi)= \begin{cases}p^{(m-1) / 2}, & \text { if } 2 \nmid m, \\ p^{(m-2) / 2} \sum_{y=0}^{p-1} \zeta_{p}^{m u y^{2} / 2}, & \text { if } 2 \mid m,\end{cases}
$$

where we've written $y$ for the variable $x_{m / 2}$. This proves (2.20) for odd $m$. Assume now that $2 \mid m$. Then

$$
\sum_{y=0}^{p-1} \zeta_{p}^{m u y^{2} / 2}=\sqrt{p}\left(\frac{m u}{p}\right) \zeta_{8}^{(1-p)(1-m)}\left(\frac{-1}{p}\right)^{m / 2}
$$


(see [2, Theorem 1.5.2, p. 26]). In view of $(2.25)-(2.26)$, it remains to prove that

$$
\left(\frac{u}{p}\right)=\left(\frac{-1}{p}\right)^{m / 2}\left(\frac{-D p^{1-m}}{p}\right) \text {. }
$$

By Lemma 2.1,

$$
N_{E / \mathbb{Q}}(\tau) \equiv-p u(\bmod q),
$$

so by $(2.18), N:=N_{E_{\mathfrak{P}} / \mathbb{Q}_{p}}(\pi)$ satifies

$$
\left(\frac{u}{p}\right)=\left(\frac{-N / p}{p}\right)
$$

By (2.15),

$$
N_{E / \mathbb{Q}}(\omega) / p \equiv N / p\left(\bmod p \mathbb{Z}_{p}\right)
$$

and so

$$
\left(\frac{u}{p}\right)=\left(\frac{-N / p}{p}\right)=\left(\frac{-\left(N_{E / \mathbb{Q}}(\omega) / p\right)^{m-1}}{p}\right)
$$

where the last equality uses the fact that $m$ is even. Let $g(x) \in \mathbb{Z}[x]$ denote the $(p$-Eisensteinian) irreducible polynomial of $\omega$ over $\mathbb{Q}$, discussed near the beginning of the proof of Lemma 2.1. Since

$$
g^{\prime}(\omega) \equiv m \omega^{m-1}\left(\bmod p \mathcal{O}_{E}\right)
$$

and $m$ is even, (2.28) yields

$$
\left(\frac{u}{p}\right)=\left(\frac{-N_{E / \mathbb{Q}}\left(g^{\prime}(\omega)\right) p^{1-m}}{p}\right)
$$

By a well-known formula for the discriminant of the basis $1, \omega, \ldots, \omega^{m-1}$ for $E$ [11. Prop. 2.4, p. 53], the "numerator" on the right side of (2.29) may be replaced by $(-1)^{(m+2) / 2} D p^{1-m}$. This proves $(2.27)$ and completes the proof of $(2.20)$ in Case 1.

Case 2: $\nu(\chi)=1, r=2 s+1, s \geq 1, q \neq 8$.

In this case, $s>1$ when $p=2$. The elements $\alpha \in \mathcal{O}_{q}^{*}$ may be written

$$
\alpha=z+z w p^{s} \quad\left(z \in \mathcal{O}_{p^{s}}^{*}, w \in \mathcal{O}_{p^{s+1}}\right),
$$

so

$$
G(\chi)=\sum_{z \in \mathcal{O}_{p^{*}}^{*}} \chi(N(z)) \zeta_{q}^{T(z)} \sum_{w \in \mathcal{O}_{p^{s+1}}} \chi\left(N\left(1+w p^{s}\right)\right) \zeta_{p^{s+1}}^{T(z w)} .
$$

Observe that

$$
N\left(1+w p^{s}\right)=1+p^{s} T(w)+\frac{1}{2} p^{2 s}\left(T(w)^{2}-T\left(w^{2}\right)\right),
$$

so since $s>1$ when $p=2$,

$$
N\left(1+w p^{s}\right)=\left(1+p^{s}+\frac{1}{2} p^{2 s}\right)^{T\left(w-p^{s} w^{2} / 2\right)} .
$$

It thus follows from the normalization (2.6) that

$$
G(\chi)=\sum_{z \in \mathcal{O}_{p}^{*} s} \chi(N(z)) \zeta_{q}^{T(z)} S(z)
$$


where

$$
S(z)=\sum_{w \in \mathcal{O}_{p^{s+1}}} \zeta_{p^{s+1}}^{T\left(z w+p^{s} w^{2} / 2-w\right)}
$$

Writing

$$
w=x+y p^{s} \quad\left(x \in \mathcal{O}_{p^{s}}, y \in \mathcal{O}_{p}\right)
$$

we have

$$
S(z)=\sum_{x} \zeta_{p^{s+1}}^{T\left(x(z-1)+x^{2} p^{s} / 2\right)} \sum_{y} \zeta_{p}^{T(y(z-1))} .
$$

The inner sum $\sum_{y}$ vanishes unless $z \equiv 1(\bmod \tau)$, in which case $\sum_{y}=p^{m}$. Thus set

$$
z=1+\sum_{i=1}^{m-1} z_{i} \tau^{i}, \quad z_{i} \in R_{p^{s}}
$$

Writing

$$
x=a+b p \quad\left(a \in \mathcal{O}_{p}, b \in \mathcal{O}_{p^{s-1}}\right),
$$

we have

$$
S(z)=p^{m} \sum_{a} \zeta_{p}^{T\left(a^{2} / 2\right)} \zeta_{p^{s+1}}^{T(a(z-1))} U(z)
$$

where

$$
U(z)=\sum_{b \in \mathcal{O}_{p^{s}-1}} \zeta_{p^{s}}^{T(b(z-1))}
$$

Writing

$$
b=\sum_{i=0}^{m-1} b_{i} \tau^{i}, \quad b_{i} \in R_{p^{s-1}},
$$

we have, by (2.31) and Lemma 2.1,

$$
U(z)=\sum_{b_{0}, \ldots, b_{m-1}} \zeta_{p^{s}}^{m p u}{\stackrel{\sum}{i=1} z_{i} z_{m-i}}_{b_{0}, \ldots, b_{m-1}} \zeta_{p^{s-1}}^{m u \sum_{i=1}^{m-1} z_{i} b_{m-i}} .
$$

Therefore $U(z)$ vanishes unless $p^{s-1}$ divides each of $z_{1}, z_{2}, \ldots, z_{m-1}$, in which case $U(z)=p^{m(s-1)}$. Thus, with

$$
z=1+p^{s-1} \sum_{i=1}^{m-1} q_{i} \tau^{i}, \quad q_{i} \in R_{p}
$$

(2.32) becomes

$$
S(z)=p^{s m} \sum_{a \in \mathcal{O}_{p}} \zeta_{p}^{T\left(a^{2} / 2\right)} \zeta_{p^{2}}^{T\left(a \sum_{i=1}^{m-1} q_{i} \tau^{i}\right)}
$$

Writing

$$
a=\sum_{i=0}^{m-1} a_{i} \tau^{i}, \quad a_{i} \in R_{p}
$$


we obtain

$$
S(z)=p^{s m} \sum_{a_{0}, \ldots, a_{m-1}} \zeta_{p}^{m a_{0}^{2} / 2+m u} \sum_{i=1}^{m-1} a_{m-i} q_{i} .
$$

Thus $S(z)$ vanishes unless $q_{1}=\cdots=q_{m-1}=0$, i.e., $S(z)$ vanishes unless $z=1$. Since

$$
S(1)=p^{s m+(m-1)} \sum_{d \in R_{p}} \zeta_{p}^{m d^{2} / 2}
$$

(2.30) yields

$$
\begin{aligned}
G(\chi) & =\zeta_{q}^{m} p^{m(s+1 / 2)} p^{m / 2-1} \sum_{d \in R_{p}} \zeta_{p}^{m d^{2} / 2} \\
& =\left(\zeta_{q} \sqrt{q}\right)^{m} p^{m / 2-1} \sum_{d \in R_{p}} \zeta_{p}^{m d^{2} / 2}
\end{aligned}
$$

By (2.3),

$$
\left(\sqrt{q} \zeta_{q}\right)^{m}= \begin{cases}G_{1}(\chi)^{m} \zeta_{8}^{-m(1-p)}, & \text { if } p>2, \\ G_{1}(\chi)^{m} \zeta_{8}^{-m}, & \text { if } p=2 .\end{cases}
$$

By [2, Theorem 1.5.2, p. 26],

$$
\sum_{d \in R_{p}} \zeta_{p}^{m d^{2} / 2}= \begin{cases}\sqrt{p}\left(\frac{m}{p}\right) \zeta_{8}^{1-p}, & \text { if } p>2, \\ 1+i^{m}=\sqrt{p}\left(\frac{p}{m}\right) \zeta_{8}^{m}, & \text { if } p=2 .\end{cases}
$$

When $p$ and $m$ are odd, the law of quadratic reciprocity gives

$$
\left(\frac{p}{m}\right)=\left(\frac{m}{p}\right) \zeta_{8}^{(1-p)(1-m)} .
$$

Combining (2.33) - (2.36), we complete the proof of (2.20) in Case 2.

Case 3: $\nu(\chi)=1, q=8$.

The elements $\alpha \in \mathcal{O}_{8}^{*}$ can be written

$$
\alpha=a+2 a b \quad\left(a \in \mathcal{O}_{2}^{*}, b \in \mathcal{O}_{4}\right),
$$

so

$$
G(\chi)=\sum_{a \in \mathcal{O}_{2}^{*}} \chi(N(a)) \zeta_{8}^{T(a)} \sum_{b \in \mathcal{O}_{4}} \chi(N(1+2 b)) \zeta_{4}^{T(a b)} .
$$

Observe that

$$
N(1+2 b)=1+2 T(b)+2\left(T(b)^{2}-T\left(b^{2}\right)\right) .
$$

Write

$$
a=1+\sum_{i=1}^{m-1} a_{i} \tau^{i}, \quad a_{i} \in R_{2},
$$

and

$$
b=\sum_{i=0}^{m-1} b_{i} \tau^{i}, \quad b_{i} \in R_{4} .
$$


We have $T(b)=m b_{0}$, and, since $m$ is odd, $T(b)^{2}=m^{2} b_{0}^{2}=b_{0}^{2}$. Also,

$$
T\left(b^{2}\right)=T\left(b_{0}^{2}\right)+T\left(2 u \sum_{i=1}^{m-1} b_{i} b_{m-i}\right) .
$$

Since $m$ is odd, $\sum b_{i} b_{m-i}$ is even, so

$$
2 T\left(b^{2}\right)=2 T\left(b_{0}^{2}\right)=2 m b_{0}^{2} .
$$

Thus (2.38) becomes

$$
N(1+2 b)=1+2 m b_{0}+2 b_{0}^{2}-2 m b_{0}^{2} .
$$

Now,

$$
T(a b)=m b_{0}+2 m u \sum_{i=1}^{m-1} a_{i} b_{m-i} .
$$

By (2.39) - (2.40), we see that (2.37) becomes

$$
\begin{aligned}
G(\chi)= & \sum_{a \in \mathcal{O}_{2}^{*}} \chi(N(a)) \zeta_{8}^{T(a)} \sum_{b_{0} \in R_{4}} \chi\left(1+2 m b_{0}+2 b_{0}^{2}(1-m)\right) \zeta_{4}^{m b_{0}} \\
& \times \sum_{b_{1}, \ldots, b_{m-1} \in R_{4}}(-1) \sum_{i=1}^{m-1} a_{i} b_{m-i}
\end{aligned}
$$

The inner sum on $b_{1}, \ldots, b_{m-1}$ vanishes unless $a_{1}, \ldots, a_{m-1}$ are all even, in which case $a=1$ and this inner sum equals $4^{m-1}$. Thus (2.41) becomes

$$
\begin{aligned}
G(\chi) & =\zeta_{8}^{m} 4^{m-1} \sum_{b_{0} \in R_{4}} \chi\left(1+2 m b_{0}+2 b_{0}^{2}(1-m)\right) \zeta_{4}^{m b_{0}} \\
& =\zeta_{8}^{m} 4^{m-1}\left\{1+\chi(3) \zeta_{4}^{m}+\chi(5) \zeta_{4}^{2 m}+\chi(7) \zeta_{4}^{3 m}\right\} .
\end{aligned}
$$

Since $\chi(5)=-1$ by $(2.5)$,

$$
\begin{aligned}
G(\chi) & =\zeta_{8}^{m} 4^{m-1}\left\{1-\chi(-1) \zeta_{4}^{m}+1-\chi(-1) \zeta_{4}^{m}\right\} \\
& =\zeta_{8}^{m} 2^{2 m-1}\left\{1-\chi(-1) \zeta_{4}^{m}\right\} \\
& =\left(\sqrt{8} \zeta_{8}^{1-\chi(-1)}\right)^{m} 2^{(m-1) / 2}\left(\frac{2}{m}\right) \\
& =G_{1}(\chi)^{m} 2^{(m-1) / 2}\left(\frac{2}{m}\right),
\end{aligned}
$$

where the last equality follows from (2.3). This proves (2.20) in Case 3, which completes the proof of (2.20) for normalized $\chi$.

We now drop the assumption that $\chi$ is normalized, and consider the general situation where $\chi$ is given by (2.7). For brevity, we rewrite (2.20) in the normalized case as

$$
G(\xi)=G_{1}(\xi)^{m} A(m),
$$

where

$$
A(m)= \begin{cases}\left(\frac{p}{m}\right)^{r} p^{(m-1) / 2}, & \text { if } 2 \nmid m \\ \zeta_{8}^{(1-p)(1-m)}\left(\frac{m}{p}\right)\left(\frac{-D p^{1-m}}{p}\right)^{r+1} p^{(m-1) / 2}, & \text { if } 2 \mid m .\end{cases}
$$


Applying the automorphism $\sigma_{\nu}$ to both sides of (2.42), we have, by (2.7) and (2.8),

$$
G(\chi)=G_{1}(\chi)^{m} \sigma_{\nu}(A(m)) .
$$

To prove $(2.20)$, it remains to show that

$$
\sigma_{\nu}(A(m))= \begin{cases}A(m), & \text { if } 2 \nmid m, \\ \left(\frac{\nu}{p}\right) A(m), & \text { if } 2 \mid m .\end{cases}
$$

If $2 \nmid m,(2.44)$ follows because $A(m) \in \mathbb{Z}$. Now suppose that $2 \mid m$ (so that $p>2$ ). Then $A(m)=n \sqrt{p} i^{(p-1)^{2} / 4}$ for some $n \in \mathbb{Z}$. Now (2.44) follows since

$$
\sqrt{p} i^{(p-1)^{2} / 4}=\sum_{x=0}^{p-1} \zeta_{p}^{x^{2}}
$$

(see [2, Theorem 1.2.4, p. 15]) and

$$
\sigma_{\nu}\left(\sum_{x=0}^{p-1} \zeta_{p}^{x^{2}}\right)=\left(\frac{\nu}{p}\right) \sum_{x=0}^{p-1} \zeta_{p}^{x^{2}} .
$$

We remark that in the case $2 \mid m,(2.20)$ can also be written

$$
G(\chi)=G_{1}(\chi)^{m-1} G_{1}(\chi \phi) p^{(m-1) / 2} \zeta_{8}^{(1-p)(1-m)}\left(\frac{-D p^{1-m}}{p}\right)^{r+1}\left(\frac{m}{p}\right), \text { if } r \geq 2,
$$

where $\phi$ is the Legendre symbol, viz., $\phi(x)=\left(\frac{x}{p}\right)$. To see this, write $\chi=\xi^{\nu}$ as in (2.7). In view of $(2.4)-(2.6), \nu(\xi \phi)=1$, so $G_{1}(\xi \phi)=G_{1}(\xi)$ by $(2.3)$; then, applying $\sigma_{\nu}$ to both sides of this equality, we obtain, by (2.8),

$$
G_{1}(\chi \phi)=\left(\frac{\nu(\chi)}{p}\right) G_{1}(\chi), \quad \text { if } r \geq 2
$$

\section{Evaluation of Kloosterman sums $K(\eta, z)$}

In the case that $E / \mathbb{Q}$ is cyclic, $p$ is an odd prime, and $m$ is a prime dividing $(p-1)$, Ye [16, Theorem 1] gave essentially the following evaluation of the Kloosterman sum $K(z)$ (defined below (1.6)):

$$
K(z)=p^{(m-1) / 2}\left(\frac{m}{p}\right)\left(\frac{-D p^{1-m}}{p}\right)^{(m+1)(r+1)} \zeta_{8}^{(1-p)(1-m)} H(z), \quad z \in R_{q}^{*}
$$

where $H(z)$ is the twisted hyper-Kloosterman sum defined by

$$
H(z)=\sum_{x_{1}, \ldots, x_{m} \in R_{q}^{*}} \psi\left(x_{2} x_{3}^{2} \cdots x_{m}^{m-1}\right) \zeta_{q}^{x_{1}+\cdots+x_{m}+z /\left(x_{1} \cdots x_{m}\right)}
$$

for any character $\psi(\bmod p)$ of order $m$. (Note that $H$ does not depend on the choice of $\psi$.) Our formulation (3.1) does not quite agree with the statement in [16. Theorem 1]. This is because when $m=2$, the factor $\eta(p)$ in [16, Theorem 1, p. $1159]$ should be corrected to read $\eta(p)^{a+1}$, which turns out to equal $\left(\frac{-D / p}{p}\right)^{r+1}$ in our notation. 
For $z \in R_{q}^{*}$ and any characters $A, B(\bmod q)$, define another twisted hyperKloosterman sum $J(A, B, z)$ by

$$
J(A, B, z)=\sum_{y_{1}, \ldots, y_{m} \in R_{q}^{*}} A\left(y_{1}\right) B\left(y_{1} \cdots y_{m}\right) \zeta_{q}^{y_{1}+\cdots+y_{m}+z /\left(y_{1} \cdots y_{m}\right)} .
$$

In (3.11) below, we give a formula for $H(z)$ in terms of the sum $J(A, B, z)$ which is valid for $r \geq 2$. The sums $H(z)$ and $J(A, B, z)$ are special cases of the general twisted hyper-Kloosterman sum

$$
K\left(A_{1}, \ldots, A_{m}, z\right):=\sum_{x_{1}, \ldots, x_{m} \in R_{q}^{*}} A_{1}\left(x_{1}\right) \cdots A_{m}\left(x_{m}\right) \zeta_{q}^{x_{1}+\cdots+x_{m}+z /\left(x_{1} \cdots x_{m}\right)},
$$

which has been evaluated for $r \geq 2$ by Evans [5. In the case $r=1$, the sum $K\left(A_{1}, \ldots, A_{m}, z\right)$ (as well as its analogue over general finite fields) was estimated by Katz 7 pp. 48-49]. When the characters $A_{1}, \ldots, A_{m}$ are all trivial, the sum $K\left(A_{1}, \ldots, A_{m}, z\right)$ reduces to the familiar hyper-Kloosterman sum $J(1,1, z)$, evaluated for $r \geq 2$ by Smith [14. (Some errors in Smith's formulations [14, Theorem 5] are corrected in [5].)

Using the Davenport-Hasse product formula (3.14), one can evaluate a sum related to $H(z)$ in the case $r=1$, namely

$$
\sum_{x_{2}, \ldots, x_{m} \in R_{p}^{*}} \psi\left(x_{2} x_{3}^{2} \cdots x_{m}^{m-1}\right) \zeta_{p}^{x_{2}+\cdots+x_{m}+z /\left(x_{2} \cdots x_{m}\right)}
$$

see Duke [4, Katz [7, p. 85] for evaluations of this sum and its analogue over finite fields.

The following lemma expresses the Kloosterman sums $K(\eta, z), J(A, B, z)$, and $H(z)$ in terms of Gauss sums $G(\chi)$; cf. Katz [7, p. 47].

Lemma 3.1. Let $z \in R_{q}^{*}$ and let $A, B, \eta$ be characters $(\bmod q)$. Then

$$
K(\eta, z)=\frac{1}{\varphi(q)} \sum_{\chi} \bar{\chi}(z) G_{1}(\chi) G(\chi \eta)
$$

(where $\chi$ runs through the $\varphi(q)$ characters $(\bmod q)$ ) and

$$
J(A, B, z)=\frac{1}{\varphi(q)} \sum_{\chi} \bar{\chi}(z) G_{1}(\chi) G_{1}(\chi A B) G_{1}(\chi B)^{m-1} .
$$

Also, if $\psi$ is a character of order $m$ (in which case $m \mid(p-1))$, then

$$
H(z)=\frac{1}{\varphi(q)} \sum_{\chi} \bar{\chi}(z) G_{1}(\chi) \prod_{j=0}^{m-1} G_{1}\left(\chi \psi^{j}\right) .
$$

Proof. For $c \in R_{q}^{*}$,

$$
\frac{1}{\varphi(q)} \sum_{\chi} \chi(c)= \begin{cases}1, & \text { if } c=1 \\ 0, & \text { if } c \neq 1\end{cases}
$$


Hence,

$$
\begin{aligned}
& \frac{1}{\varphi(q)} \sum_{\chi} \bar{\chi}(z) G_{1}(\chi) G(\chi \eta) \\
& \quad=\frac{1}{\varphi(q)} \sum_{\chi} \bar{\chi}(z) \sum_{y \in R_{q}^{*}} \chi(y) \zeta_{q}^{y} \sum_{\alpha \in \mathcal{O}_{q}^{*}} \chi(N(\alpha)) \eta(N(\alpha)) \zeta_{q}^{T(\alpha)} \\
& \quad=\sum_{\alpha \in \mathcal{O}_{q}^{*}} \eta(N(\alpha)) \zeta_{q}^{T(\alpha)+z / N(\alpha)}=K(\eta, z) .
\end{aligned}
$$

This proves (3.4). The proofs of (3.5) and (3.6) are completely analogous.

Theorem 3.2 below extends Ye's evaluation (3.1) of $K(1, z)$ for $r \geq 2$ by showing that for any odd prime $p,(3.1)$ holds for all (not necessarily prime) values of $m$ dividing $p-1$. More generally, for $r \geq 2$ and any prime $p \geq 2$, Theorem 3.2 gives an evaluation of $K(\eta, z)$ for all $m$ (not necessarily prime or a divisor of $p-1$ ), in terms of the sum $J$ defined in (3.3). For evaluations of $J$, see [14, [5].

The case $r=1$ will be considered in Theorem 3.3.

Theorem 3.2. Let $r \geq 2$ and $z \in R_{q}^{*}$. Let $\eta$ be any character $(\bmod q)$ and let $\phi$ denote the Legendre symbol, viz., $\phi(x)=\left(\frac{x}{p}\right)$. Then

$$
K(\eta, z)=p^{(m-1) / 2}\left(\frac{p}{m}\right)^{r} J(1, \eta, z), \quad \text { if } 2 \nmid m,
$$

and

$$
K(\eta, z)=p^{(m-1) / 2}\left(\frac{m}{p}\right)\left(\frac{-D p^{1-m}}{p}\right)^{r+1} \zeta_{8}^{(1-p)(1-m)} J(\phi, \eta, z), \quad \text { if } 2 \mid m,
$$

where $J(A, B, z)$ is defined by (3.3). Moreover, for every odd prime $p$ and every $m$ dividing $(p-1),(3.1)$ holds.

Proof. If $m$ is odd, then by (3.4) and (2.20),

$$
\begin{aligned}
K(\eta, z) & =p^{(m-1) / 2}\left(\frac{p}{m}\right)^{r} \frac{1}{\varphi(q)} \sum_{\chi} \bar{\chi}(z) G_{1}(\chi) G_{1}(\chi \eta)^{m} \\
& =p^{(m-1) / 2}\left(\frac{p}{m}\right)^{r} J(1, \eta, z),
\end{aligned}
$$

where the last equality follows from (3.5) with $A=1, B=\eta$. This proves (3.7).

If $m$ is even, then by (3.4) and (2.45),

$$
\begin{aligned}
& K(\eta, z)=p^{(m-1) / 2}\left(\frac{-D p^{1-m}}{p}\right)^{r+1}\left(\frac{m}{p}\right) \zeta_{8}^{(1-p)(1-m)} \frac{1}{\varphi(q)} \\
& \quad \times \sum_{\chi} \bar{\chi}(z) G_{1}(\chi) G_{1}(\chi \eta \phi) G_{1}(\chi \eta)^{m-1} .
\end{aligned}
$$

By (3.5) with $A=\phi, B=\eta$, this proves (3.8).

Next let $p$ be an odd prime $\equiv 1(\bmod m)$. It remains to prove $(3.1)$.

Let $\psi$ be a character $(\bmod p)$ of order $m$ and write $\chi=\xi^{\nu}$ as in (2.7). In view of (2.4) - (2.6), $\nu\left(\xi \psi^{i}\right)=1$ for all $i$, so that by $(2.3), G_{1}\left(\xi \psi^{i}\right)=G_{1}(\xi)$ for all $i$. Thus

$$
\prod_{i=0}^{m-1} G_{1}\left(\xi \psi^{i}\right)=G_{1}(\xi)^{m}
$$


Since $\nu=\nu(\chi)$ is relatively prime to $p-1$, it follows that $\nu$ is relatively prime to $m$. Hence, applying $\sigma_{\nu}$ to both sides of (3.9), we obtain, by (2.8),

$$
\prod_{i=0}^{m-1} G_{1}\left(\chi \psi^{i}\right)=\left(\frac{\nu}{p}\right)^{m-1} G_{1}(\chi)^{m}
$$

Thus, by (2.46),

$$
\prod_{i=0}^{m-1} G_{1}\left(\chi \psi^{i}\right)= \begin{cases}G_{1}(\chi)^{m}, & \text { if } 2 \chi m \\ G_{1}(\chi)^{m-1} G_{1}(\chi \phi), & \text { if } 2 \mid m .\end{cases}
$$

Putting (3.10) in (3.6) and then using (3.5), we see that for $r \geq 2$,

$$
H(z)= \begin{cases}J(1,1, z), & \text { if } 2 \nmid m \\ J(\phi, 1, z), & \text { if } 2 \mid m\end{cases}
$$

Set $\eta=1$ in (3.7) - (3.8) and make the substitution (3.11). Then using (2.36) for odd $m$ and noting that $\left(\frac{p}{m}\right)=1($ since $p \equiv 1(\bmod m))$, we obtain $(3.1)$.

For the remainder of this section, let $r=1$. Then

$$
K(\eta, z)=\sum_{\alpha \in \mathcal{O}_{p}^{*}} \eta(N(\alpha)) \zeta_{p}^{T(\alpha)+z / N(\alpha)} .
$$

By (2.11), we can write

$$
\alpha=a+a_{1} \tau+\cdots+a_{m-1} \tau^{m-1} \quad\left(a \in R_{p}^{*}, a_{i} \in R_{p}\right) .
$$

Then $N(\alpha)=a^{m}$ and $T(\alpha)=m a$, so that

$$
K(\eta, z)=p^{m-1} \sum_{a=1}^{p-1} \eta^{m}(a) \zeta_{p}^{m a+z / a^{m}}, \quad \text { when } r=1 .
$$

In Theorem 3.3 below, we extend Ye's result (3.1) for $r=1$ by showing that for any odd prime $p,(3.1)$ holds for all $m$ dividing $p-1$.

We will need the product formula of Davenport-Hasse [2, Theorem 11.3.5, p. 355] for the Gauss sums

$$
\gamma(\chi):=\sum_{a=1}^{p-1} \chi(a) \zeta_{p}^{a}
$$

namely,

$$
\bar{\chi}^{m}(m) \gamma\left(\chi^{m}\right)=\prod_{j=0}^{m-1} \gamma\left(\chi \psi^{j}\right) / \prod_{j=1}^{m-1} \gamma\left(\psi^{j}\right),
$$

where $\psi$ is a character $(\bmod p)$ of order $m$ (so that $m \mid(p-1))$. Note that $\gamma(\chi)$ is the Gauss sum $G_{1}(\chi)$ in the case $r=1$. It is not difficult to show that for $p>2$,

$$
\prod_{j=1}^{m-1} \gamma\left(\psi^{j}\right)=p^{(m-1) / 2}\left(\frac{m}{p}\right) \zeta_{8}^{(1-p)(m-1)} ;
$$


see [2, p. 352]. Substituting (3.15) into (3.14), we obtain the following version of the Davenport-Hasse formula, when $p>2, m \mid(p-1)$ :

$$
\bar{\chi}^{m}(m) \gamma\left(\chi^{m}\right) p^{(m-1) / 2}\left(\frac{m}{p}\right) \zeta_{8}^{(1-p)(m-1)}=\prod_{j=0}^{m-1} \gamma\left(\chi \psi^{j}\right) .
$$

Theorem 3.3. Let $r=1$ and $z \in R_{p}^{*}$, where $p$ is an odd prime. Then (3.1) holds for every $m$ dividing $(p-1)$.

Proof. Let $\psi$ be a character $(\bmod p)$ of order $m$. Since by $(2.19)$,

$$
G(\chi)=p^{m-1} \bar{\chi}^{m}(m) \gamma\left(\chi^{m}\right)
$$

if follows from (3.16) that

$$
G(\chi)=p^{(m-1) / 2}\left(\frac{m}{p}\right) \zeta_{8}^{(1-p)(1-m)} \prod_{j=0}^{m-1} \gamma\left(\chi \psi^{j}\right) .
$$

Substituting (3.17) into (3.4) with $\eta=1$, we obtain

$$
K(z)=p^{(m-1) / 2}\left(\frac{m}{p}\right) \zeta_{8}^{(1-p)(1-m)} H(z),
$$

by (3.6). This completes the proof, as (3.18) is the same as (3.1) in the case $r=1$.

\section{A product formula for Gauss sums $G(\chi)$}

In Theorem 4.1 below, we give a product formula for the Gauss sums $G(\chi)$, which in the case $m=r=1$ reduces to the Davenport-Hasse product formula (3.16).

Theorem 4.1. Let $p$ be an odd prime and let $\psi$ be a character $(\bmod p)$ of order $\ell$ (so that $\ell \mid(p-1))$. Let $\chi$ be any character $(\bmod q)$. Then if $r \geq 2$,

$$
\bar{\chi}^{\ell m}(\ell) G\left(\chi^{\ell}\right) p^{(\ell-1)(r m+m-1) / 2} C(\chi)=\prod_{j=0}^{\ell-1} G\left(\chi \psi^{j}\right),
$$

where $C(\chi) \in\{ \pm 1, \pm i\}$ is defined by

$$
C(\chi):=\left\{\begin{array}{l}
\left(\frac{\nu(\chi)}{p}\right)^{\ell-1}, \quad \text { if } 2 \nmid m, 2 \mid r, \\
\zeta_{8}^{(1-p)(\ell-1)}\left(\frac{\ell}{p}\right)\left(\frac{m}{p}\right)^{\ell-1}\left(\frac{\nu(\chi)}{p}\right)^{(\ell-1)(m-1)}, \text { if } 2 \nmid r, \\
\zeta_{8}^{(1-p)(1-m)(\ell-1)}\left(\frac{\ell}{p}\right)\left(\frac{m}{p}\right)^{\ell-1}\left(\frac{-D p^{1-m}}{p}\right)^{\ell-1}\left(\frac{\nu(\chi)}{p}\right)^{\ell-1}, \quad \text { if } 2|m, 2| r,
\end{array}\right.
$$

with $\nu(\chi)$ defined by (2.4) and (2.6). If in the case $r=1$, we define the (previously undefined) expression $\nu(\chi)$ by setting $\nu(\chi)=1$, then (4.1) also holds when $r=1$, provided that $(m, \ell)=1$.

Proof. We first consider the case $r \geq 2$. If $\chi$ is nonprimitive, then both sides of (4.1) vanish by Theorem 2.2. Assume therefore that $\chi$ is primitive.

First suppose that $\chi$ is normalized, i.e., $\nu(\chi)=1$. In this case $\chi \psi^{j}$ is normalized, i.e., $\nu\left(\chi \psi^{j}\right)=1$, for each $j$. Hence by (2.20) and (2.3),

$$
G(\chi)=G\left(\chi \psi^{j}\right), \quad \text { for all } j .
$$


Choose $b$ relatively prime to $q(p-1)$ such that $b \equiv \ell(\bmod q)$, and define $c$ by $b c \equiv 1(\bmod q(p-1))$. We claim that

$$
\sum_{\alpha \in \mathcal{O}_{q}^{*}} \chi^{b}(N(\alpha)) \zeta_{q}^{T(\alpha b)}=\sum_{\alpha \in \mathcal{O}_{q}^{*}} \chi^{\ell}(N(\alpha)) \zeta_{q}^{T(\alpha \ell)} .
$$

To verify (4.4), apply $\sigma_{c}$ to both sides to obtain $G(\chi)$ on the left and $G\left(\chi^{c \ell}\right)$ on the right; then note that $\nu(\chi)=\nu\left(\chi^{c \ell}\right)=1$, so that $G(\chi)=G\left(\chi^{c \ell}\right)$ by $(2.20)$ and (2.3).

We can rewrite (4.4) as

$$
\sigma_{b} G(\chi)=\bar{\chi}^{\ell m}(\ell) G\left(\chi^{\ell}\right)
$$

In view of (4.3) and (4.5), the proposed equality (4.1) is equivalent to

$$
\sigma_{b}(G(\chi)) p^{(\ell-1)(r m+m-1) / 2} C(\chi)=G(\chi)^{\ell} .
$$

By (2.42) and by (2.44) with $\nu=b$, the left side of (4.6) equals

$$
\sigma_{b}\left(G_{1}(\chi)^{m}\right)\left(\frac{\ell}{p}\right)^{m+1} A(m) p^{(\ell-1)(r m+m-1) / 2} C(\chi),
$$

while the right side of (4.6) equals

$$
G_{1}(\chi)^{m \ell} A(m)^{\ell}
$$

Thus (4.6) (and hence (4.1)) is equivalent to

$$
C(\chi)=A(m)^{\ell-1}\left(\frac{\ell}{p}\right)^{m+1} p^{(1-\ell)(r m+m-1) / 2} G_{1}(\chi)^{m \ell} / \sigma_{b}\left(G_{1}(\chi)^{m}\right) .
$$

Substitute the value of $G_{1}(\chi)$ given by (2.3) into (4.7) to see, after a tedious calculation, that (4.7) is equivalent to $(4.2)$ when $\nu(\chi)=1$. This completes the proof of (4.1) for $r \geq 2$ when $\nu(\chi)=1$. To prove (4.1) for $r \geq 2$ and general $\nu(\chi)$, first write down (4.1) with $\xi$ in place of $\chi$ (in the notation of (2.7)). Then, applying $\sigma_{\nu}$ to both sides, we obtain (4.1). This completes the proof of the theorem in the case $r \geq 2$.

Now let $r=1$ and assume that $(m, \ell)=1$. It remains to prove

$$
\bar{\chi}^{\ell m}(\ell) G\left(\chi^{\ell}\right) p^{(\ell-1)(m-1 / 2)} C=\prod_{j=0}^{\ell-1} G\left(\chi \psi^{j}\right),
$$

where

$$
C=\zeta_{8}^{(1-p)(\ell-1)}\left(\frac{\ell}{p}\right)\left(\frac{m}{p}\right)^{\ell-1}
$$

Since, by (2.19),

$$
G(\chi)=\bar{\chi}^{m}(m) p^{m-1} \gamma\left(\chi^{m}\right)
$$

for every character $\chi(\bmod p)$, we have in particular,

$$
G\left(\chi \psi^{j}\right)=\bar{\chi}^{m}(m) \bar{\psi}(m)^{m j} p^{m-1} \gamma\left(\chi^{m} \psi^{m j}\right)
$$

for all $j$, and

$$
\bar{\chi}^{\ell m}(\ell) G\left(\chi^{\ell}\right)=\bar{\chi}^{\ell m}(m \ell) p^{m-1} \gamma\left(\chi^{m \ell}\right) .
$$


Because $(m, \ell)=1$, it follows that $\psi^{m j}$ runs through the same characters as $\psi^{j}$ does when $j$ runs through $0,1,2, \ldots, \ell-1$. Thus, by (4.10),

$$
\prod_{j=0}^{\ell-1} G\left(\chi \psi^{j}\right)=\bar{\chi}^{m \ell}(m) p^{\ell(m-1)}\left(\frac{m}{p}\right)^{\ell-1} \prod_{j=0}^{\ell-1} \gamma\left(\chi^{m} \psi^{j}\right) .
$$

By (3.16) with $m=\ell$,

$$
\prod_{j=0}^{\ell-1} \gamma\left(\chi^{m} \psi^{j}\right)=\bar{\chi}^{m \ell}(\ell) p^{(\ell-1) / 2} \gamma\left(\chi^{m \ell}\right)\left(\frac{\ell}{p}\right) \zeta_{8}^{(1-p)(\ell-1)} .
$$

Multiplying (4.13) by (4.12) and then dividing the resulting equality by (4.11), we obtain (4.8).

\section{REFERENCES}

[1] J. Arthur and L. Clozel, Simple Algebras, Base Change, and the Advanced Theory of the Trace Formula, Annals of Math. Studies, No. 120, Princeton University Press, Princeton, 1989. MR 90m:22041]

[2] B. C. Berndt, R. J. Evans, and K. S. Williams, Gauss and Jacobi Sums, Wiley-Interscience, N.Y., 1998. MR 99d:11092

[3] H. Davenport and H. Hasse, Die Nullstellen der Kongruenzzetafunktionen in gewissen zyklischen Fällen, J. Reine Angew. Math. 172(1934), 151-182.

[4] W. Duke, On multiple Salié sums, Proc. Amer. Math. Soc. 114(1992), 623-625. MR 92f: 11113

[5] R. J. Evans, Twisted hyper-Kloosterman sums over finite rings of integers, Proceedings of the Millennial Conference on Number Theory, University of Illinois (May 21-26, 2000), A K Peters, Natick, MA, to appear in 2001.

[6] T. Funakura, A generalization of the Chowla-Mordell theorem on Gaussian sums, Bull. London Math. Soc. 24(1992), 424-430. MR 93e:11094

[7] N. Katz, Gauss Sums, Kloosterman Sums, and Monodromy Groups, Annals of Math. Studies, No. 116, Princeton University Press, Princeton, 1988. MR 91a:11028

[8] N. Koblitz, p-adic Numbers, p-adic Analysis, and Zeta-Functions, Springer-Verlag, N. Y., 1977. MR 57:5964]

[9] J.-L. Mauclaire, Sommes de Gauss modulo $p^{\alpha}$ I, Proc. Jap. Acad. Ser. A 59(1983), 109-112. MR 85f:11062a

[10] J.-L. Mauclaire, Sommes de Gauss modulo $p^{\alpha}$ II, Proc. Jap. Acad. Ser. A 59(1983), 161-163. MR 85f:11062a

[11] W. Narkiewicz, Elementary and Analytic Theory of Numbers, Springer-Verlag, Berlin and PWN-Polish Scientific Publishers, Warsaw, 1990. MR 91h:11107

[12] R. Odoni, On Gauss sums $\left(\bmod p^{n}\right), n \geq 2$, Bull. London Math. Soc. 5(1973), 325-327. MR 48:6020

[13] H. Salié, Über die Kloostermanschen Summen $S(u, v ; q)$, Math. Z. 34(1932), 91-109.

[14] R. Smith, On n-dimensional Kloosterman sums, J. Number Theory 11 (1979), 324-343. MR 80i: 11052

[15] L. C. Washington, Introduction to Cyclotomic Fields, 2nd edition, Springer-Verlag, N. Y., 1997. MR 97h:11130

[16] Y. Ye, A hyper-Kloosterman sum identity, Science in China (Series A) 41(1998), 1158-1162. MR 99m:11094

[17] Y. Ye, The lifting of an exponential sum to a cyclic algebraic number field of prime degree, Trans. Amer. Math. Soc. 350(1998), 5003-5015. MR 99b:11092

[18] Y. Ye, Personal communication, November, 1999.

Department of Mathematics, University of California at San Diego, La Jolla, CalIFORNIA 92093-0112

E-mail address: revans@ucsd.edu 